\title{
The Impact of an Automated Patient Digital Engagement Platform on Revisit Reduction
}

\author{
Adam Beck", MHS; Caroline Robinson", MBA \\ LifeBridge Health - Sinai Hospital of Baltimore, Baltimore, MD, United States \\ *all authors contributed equally
}

Corresponding Author:

Adam Beck, MHS

LifeBridge Health - Sinai Hospital of Baltimore

$2401 \mathrm{~W}$ Belvedere Ave

Baltimore, MD

United States

Phone: 4106019813

Email: abeck@lifebridgehealth.org

\section{Abstract}

Background: Revisits within 30 days to an emergency department (ED), observation care unit, or inpatient setting following patient discharge continues to be a challenge, especially in urban settings. In addition to the consequences for the patient, these revisits have a negative impact on a health system's finances in a value based care or global budget environment.

Objective: The objective was to evaluate the effectiveness of a customized automated digital patient engagement application (GetWell Loop) to prevent 30-day revisits after home discharge from an ED or hospital inpatient setting.

Methods: The LifeBridge Health Innovation Team collaborated with the GetWell Network to customize their patient engagement platform (GetWell Loop) with automated check-in questions and resources. An application link was emailed to adult patients discharged home from the ED. A retrospective study of ED visits for patients treated for general medicine and cardiology conditions (accounting for 24\% of our adult ED discharges) between August 1, 2018, and December 31, 2018, was conducted using CRISP, Maryland's state-designated health information exchange. We used this database to identify the index visits that experienced an emergency department visit, inpatient admission, or observation stay at any Maryland facility within 30 days of discharge. We also used data within GetWell Loop to track patient activation and engagement. The primary endpoint was a comparison of ED patients that experienced a 30-day revisit and who did or did not activate their GetWell Loop account. Secondary end points included overall activation rate and the rate of engagement as measured by the number of logins, alerts, and comments generated by patients through the platform. Statistical significance was calculated using the Fisher's exact test with a $P<.05$.

Results: ED discharges who were treated for general medicine conditions $(n=787)$ and activated their GetWell Loop account experienced a 30 -day revisit rate of $18.9 \%$ compared to $25.2 \%$ who did not activate their account $(P=.06)$. For patients treated for cardiology conditions $(\mathrm{n}=722), 10.5 \%$ of patients who activated their GetWell account experienced a 30-day revisit compared to $17.4 \%$ not activating their account $(P=.02)$. During the course of this study, $26 \%$ of patients receiving an invite to use the digital platform activated their account $(n=1652)$ logged in a total of 4006 times, generated 734 alerts, and submitted 297 open ended comments/questions.

Conclusions: These results indicate the potential value of digital health platforms to improve 30-day revisit rates. The strongest impact was observed amongst cardiology patients where the revisit rate is $39.8 \%$ lower for patients using GetWell Loop compared to general medicine patients where the relative difference is $25.2 \%$. The results also indicate patients are willing to utilize a digital platform postdischarge to proactively engage in their own care. We attempted to control for potential selection bias that may impact this analysis given patient adoption and use of a digital platform by looking for differences in the subpopulations who did and did not activate the platform. LifeBridge Health is proving healthcare systems can leverage automated mobile platforms to successfully impact clinical outcomes at scale without compromising customer service and patient experience.

(iproc 2019;5(1):e15091) doi: 10.2196/15091 
Edited by R Palacholla; this is a non-peer-reviewed article. Submitted 18.06.19; accepted 14.08.19; published 02.10.19.

Please cite as:

Beck A, Robinson C

The Impact of an Automated Patient Digital Engagement Platform on Revisit Reduction iproc 2019;5(1):e15091

URL: http://www.iproc.org/2019/1/e15091/

doi: $\underline{10.2196 / 15091}$

PMID:

(C)Adam Beck, Caroline Robinson. Originally published in Iproceedings (http://www.iproc.org), 02.10.2019 This is an open-access article distributed under the terms of the Creative Commons Attribution License (https://creativecommons.org/licenses/by/4.0/), which permits unrestricted use, distribution, and reproduction in any medium, provided the original work, first published in Iproceedings, is properly cited. The complete bibliographic information, a link to the original publication on http://www.iproc.org/, as well as this copyright and license information must be included. 